\title{
Autofrettage process: finite element simulation and experimental validation
}

\author{
R. Drummond \& T. Comlekci \\ Weir Advanced Research Centre, Department of Mechanical and \\ Aerospace Engineering, University of Strathclyde, UK
}

\begin{abstract}
Autofrettage of benchmark cylindrical pressure vessels were carried out for two types of low alloy steels. Non-linear finite element studies were first carried out in order to find a safe autofrettage pressure with known yield strength and ultimate tensile strength material property values from tensile tests. The residual stresses and strains were predicted with the non-linear finite element analysis (FEA) when the vessel was unloaded. The pressure vessels were strain gaged internally and externally to validate the autofrettage FEA simulation. The response of the internal strain gages under high hydrostatic pressure was not known therefore strain gaged hydrostatic test pieces were used during the experiments. Through measurement of the strain response with relation to the hydrostatic pressure applied during the autofrettage experiment, hydrostatic correction factors were found. These correction factors were applied to the pressure vessels' internal strain gage readings and compared with that of FEA. Strains during loading showed good agreement, although measuring the residual strain levels experimentally was found to be prone to error due to the variability of the material properties and the accuracy of internal strain gage measurements subject to very high hydrostatic pressure loading.

Keywords: autofrettage, validation, strain gage, hydrostatic correction, finite element analysis.
\end{abstract}

\section{Introduction}

Autofrettage is an industrial process which over-pressurizes a pressure component to induce a specific level of plastic strain. This plasticity leads to the induction of a compressive residual stress on the internal surface of the component when the 


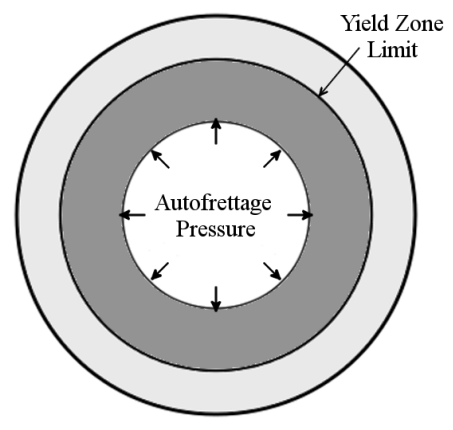

Figure 1: Profile of a thick walled cylinder after autofrettage.

autofrettage pressure is removed. Compressive residual stress fields on the surface of components have been shown to be beneficial to the fatigue life of a range of components [1,2].

Hydraulic autofrettage consists of the over pressurization of a component to cause partial plastic yielding within it. This plastic inner surface leads to compressive stresses from the surrounding elastic region. Manipulation of these compressive residual stresses can be used to improve the fatigue life of the component as these compressive residual stresses will need to be overcome when the component is loaded during operation.

\section{Modeling fatigue}

When attempting to predict the fatigue life of a component it is important to determine an approximate life cycle range, since the dominance of plastic or elastic material behavior shifts from that of low to high cycle fatigue respectively. Fatigue failure is generally divided into low and high cycle ranges, with the cut off of the low cycle fatigue range being around $10^{4}$ cycles [3]. Below this threshold plastic strain behavior dominates the fatigue damage, leading to the Strain-Based fatigue approach. The components tested here lie within the high to very high cycle fatigue range, so then the Stress-Based approach is more appropriate.

The Stress-based fatigue life approach relies on the use of simplifying the load cycle to either a single simple sinusoidal cycle, or that of a series of simple sinusoidal cycles. It is assumed that by comparing the stresses at the maximum and minimum points of the assumed cycle, an alternating stress intensity can be calculated.

Tensile testing machines can be used to obtain experimental fatigue data on similar material. When these data are plotted as stress amplitude against the number of cycles till failure on a log-log scale, the resulting $\mathrm{S}-\mathrm{N}$ curve can be used to evaluate an approximate number of cycles to failure from the alternating stress 


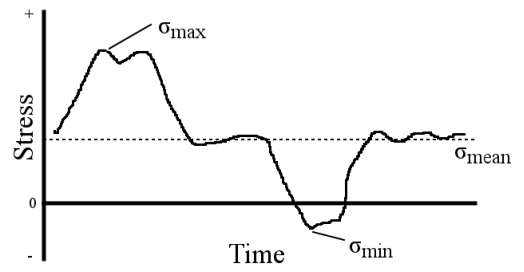

Figure 2: An example of real world loading.

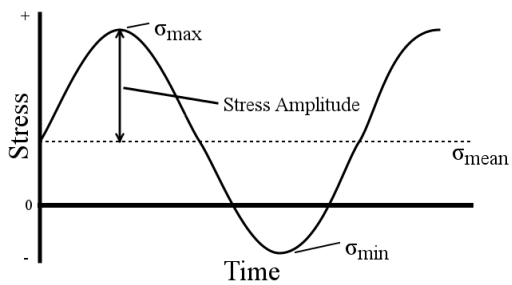

Figure 3: Simplified loading used for calculation.

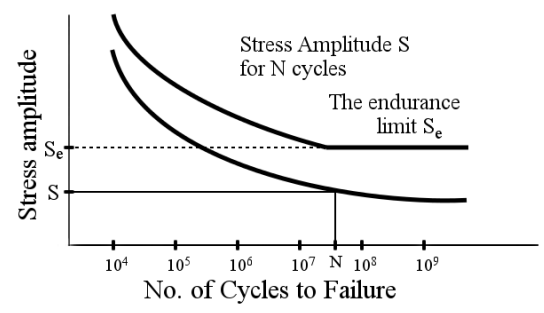

Figure 4: An example of a plot of stress amplitude against number of cycles to failure curve on a log-log scale, commonly known as an S-N curve.

intensity and hence an approximate fatigue life. Since the S-N data is based upon a $\log$-log relationship, then any slight decrease in the alternating stress intensity will result in dramatic increases in the fatigue life.

\section{Material models}

True stress-strain curves cannot be input directly into FEA simulations due to the number of data points, therefore approximations are used. Two main variations of material model were used during the FEA simulation. Both material models were based upon a kinematic hardening model to model the Bauschinger effect. The first model type was a bi-linear model, while the other was a multi-linear model. A Bi-linear model approximates the stress-strain curve into 2 intersecting straight lines with different gradients; the first describes the elastic region while the second line describes the plastic region. A multi-linear model approximates a stress-strain curve in a similar way, however retains more information about the initial plastic strain by using more intersecting straight lines.

The multi-linear kinematic model that was used is derived from the use of the ASME BPVC KD231.4 procedure code [4], that describes the calculation of an approximation of the true stress-strain curve for a range of ductile metals from their material properties. From this procedure a number of data points were selected, to 
coincide with the limit of maximum allowable material property data inputs by the FEA software used. The selection method for these points was tested to reduce the level of information lost concerning the plastic behavior to a minimum.

During the experiment design phase, material properties from previous material tests were used. However later, during comparison of FEA with the strain readings, new material data used during the validation process was obtained from the same material block that was used to create both pressure vessels in all three co-ordinate directions. From these tests, a slight anisotropy was found to exist for both types of steel that were used. Material from both pressure vessels was seen to vary in the different directions by as much as $4.9 \%$ in their yield stress and UTS values.

\section{The experiment}

A pressure vessel was designed to provide a simplified version of the geometry present within a complex pressure vessel under investigation. The main aims of the experiment were:

- The validation of the FEA method and material models used to simulate the autofrettage process and the residual stresses induced.

- Investigate and quantify the hydrostatic pressure effect on strain gages used to measure the residual stresses induced during autofrettage.

The initial design of the benchmark pressure vessels was based on FEA and material models currently used to simulate the autofrettage process to find the balance between measurement of sufficiently large residual stresses within the vessels through the use of pressures representative of those used during autofrettage, whilst also restricting the propagation of plasticity to an unsafe level through the vessel wall. To fully measure the hydrostatic effect on the strain gages up to pressures representative within autofrettage currently being carried out, the pressures being designed to were set to comparable values during the design process. The geometry of the design was then driven so as to provide several hundred microstrains residual strain within the axial and hoop directions with the highest yield case scenario material model; whilst also not allowing plasticity to penetrate through $50 \%$ of the wall thickness with the lowest yield case material.

Strain gages were positioned both inside and outside the same axial position on the thick walled cylinder section of the pressure vessels. The pressure vessel was designed in such a way that the central section had a thinner section and therefore would plastically deform here, whilst leaving the rest of the vessel to deform elastically. Since this central section was designed to act similarly to a thick walled cylinder, the plastic deformation would remain relatively uniform axially meaning that the inaccurate placement of the gages would not effect their readings greatly.

The pressure vessels were designed to behave as thick walled cylinders at their thinnest section to simplify the comparisons with analytical and FEA simulations, as well as reduce the possible errors induced through strain gage placement. The 
analytical theory behind the autofrettage of thick walled cylinders has been well established [5].

Instantaneous data acquisition was carried out throughout the autofrettage procedure as well as 3 subsequent loading and unloading steps. Theses loadsteps were of comparable pressures seen within components and used to gather further experimental data to have an additional data set for validation of calculated hydrostatic correction factors.

\section{Residual stress measurement}

Traditionally residual stress measurements are taken either before or after an industrial process has taken place. These techniques fall into one of either two main method types [6]:

- Diffraction methods that require the use of high energy electromagnetic waves or particles impacting on the site of interest and using their subsequent diffraction patterns to infer the strains present within the material.

- Relaxation methods requires the removal of material through a variety of methods, in such a way as to induce as little residual stress as possible. Measurements are taken of the surrounding material to see how it relaxes, thereby inferring the levels of strain that were present within the material that was removed.

Neither of these methods measures the instantaneous residual strains and therefore stresses. To attempt to measure the instantaneous residual strain induction throughout the process, strain gage rosettes were placed in a number of locations both internally and externally. These were then used to measure the strain values induced on the surface of the material.

\section{Strain gages}

Strain gages work upon the principal that deformations in a conductive material will cause a proportional change in the electric resistance through it. By folding a thin wire back on itself repeatedly, the effect of these deformations in one direction will then dominate this resistance change. Strains measured in this way will measure both the elastic and plastic strains present, also known as the total mechanical strain. In the case of hydrostatic pressure, deformation perpendicular to this primary direction occurs, also causing a change in the electrical resistance. To quantify this effect hydrostatic test pieces were suspended within the hydraulic fluid used to pressurize the pressure vessels and the hydrostatic response of the gages measured. Previous attempts were deemed to have a rather large error margin $[7,8]$.

By comparing the presumed hydrostatic response of the test pieces with the measured response of the strain gages, a model of the gage response with respect to hydrostatic pressure was found. Two different conversion factors were calculated, 


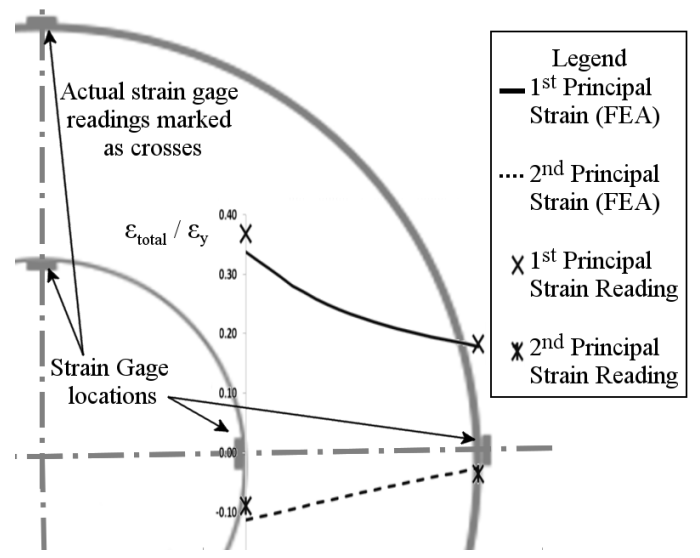

Figure 5: Mat B through thickness strain comparison. The residual strain gage readings can be seen here overlayed onto the FEA through thickness results.

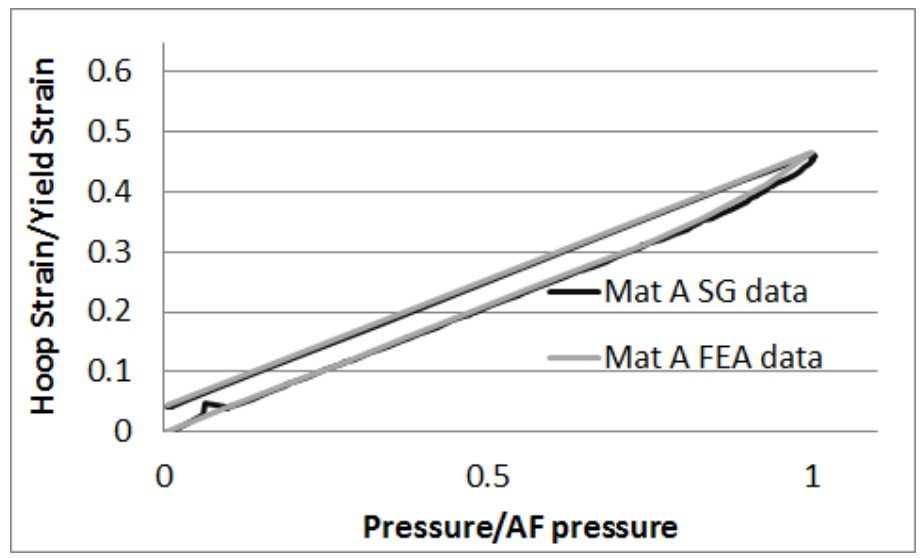

Figure 6: Material A External Gage Comparison.

one for the Autofrettage and another for the Load cycling data sets. Comparisons between them showed a reasonably small variation in conversion factor parameter values. This subsequently led to a conversion method that was then used for strain gages under hydrostatic pressure.

A singular strain gage can only measures the strains within a singular direction. To get the full strain profile, at least 3 strain gage readings should be taken. For ease of use these three gages can be readily purchased in the form of a strain gage rosette, at set orientations from one another. When these different orientations are known, then the principal strains and their direction in relation to one of the gages can be calculated. Since the hydrostatic strain gage effect acts upon all strain gages 
Table 1: Percentage errors comparing FEA and Material A Strain Gage Results.

\begin{tabular}{|l|c|c|c|}
\hline \multicolumn{1}{|c|}{ Case } & Internal Gage & $1^{\text {st }}$ External Gage & $2^{\text {nd }}$ External Gage \\
\hline $\begin{array}{l}\text { During loading Min. } \\
\text { error }\end{array}$ & $0.5 \%$ & $0.1 \%$ & $0.6 \%$ \\
\hline $\begin{array}{l}\text { During loading Max. } \\
\text { error }\end{array}$ & $2.9 \%$ & $2.5 \%$ & $2.5 \%$ \\
\hline $\begin{array}{l}\text { At Autofrettage Pres- } \\
\text { sure }\end{array}$ & $2.6 \%$ & $1.9 \%$ & $0.6 \%$ \\
\hline Unloaded \% error & $13.9 \%$ & $16.7 \%$ & $12.3 \%$ \\
\hline $\begin{array}{l}\text { Unloaded error } \\
\text { (microstrain) }\end{array}$ & 59.6 & 31.4 & 29.8 \\
\hline
\end{tabular}

Table 2: Percentage Errors comparing FEA and Material B Strain Gage Results.

\begin{tabular}{|l|c|c|c|}
\hline \multicolumn{1}{|c|}{ Case } & Internal Gage & $1^{\text {st }}$ External Gage & $2^{\text {nd }}$ External Gage \\
\hline $\begin{array}{l}\text { During loading Min. } \\
\text { error }\end{array}$ & $1.8 \%$ & $0.8 \%$ & $0.6 \%$ \\
\hline $\begin{array}{l}\text { During loading Max. } \\
\text { error }\end{array}$ & $3.3 \%$ & $4.2 \%$ & $2.4 \%$ \\
\hline $\begin{array}{l}\text { At Autofrettage Pres- } \\
\text { sure }\end{array}$ & $3.8 \%$ & $0.1 \%$ & $0.3 \%$ \\
\hline Unloaded \% error & $8.4 \%$ & $2.0 \%$ & $11.2 \%$ \\
\hline $\begin{array}{l}\text { Unloaded error } \\
\text { (microstrain) }\end{array}$ & 44.2 & 7.3 & 28.6 \\
\hline
\end{tabular}

within a rosette equally, hydrostatic correction was carried out before conversion of the strain gage readings to principal strains. Due to the shape of the pressure vessels, the directions of the principal strains at the locations of the strain gages aligned themselves pretty closely with that of the hoop and axial directions. The slight anisotropy of the material may have altered this direction slightly, however the effect was deemed negligible.

Strain gages were placed both internally and externally at the same points radially and axially to compare both "corrected" internal strain gage readings and FEA, as well as raw external strain gage data. Although not measuring any residual strain directly, since residual strains average over the entire component to zero, the effect of the internal "misfit" of plastic material was still detectable to a lesser degree externally [9]. 


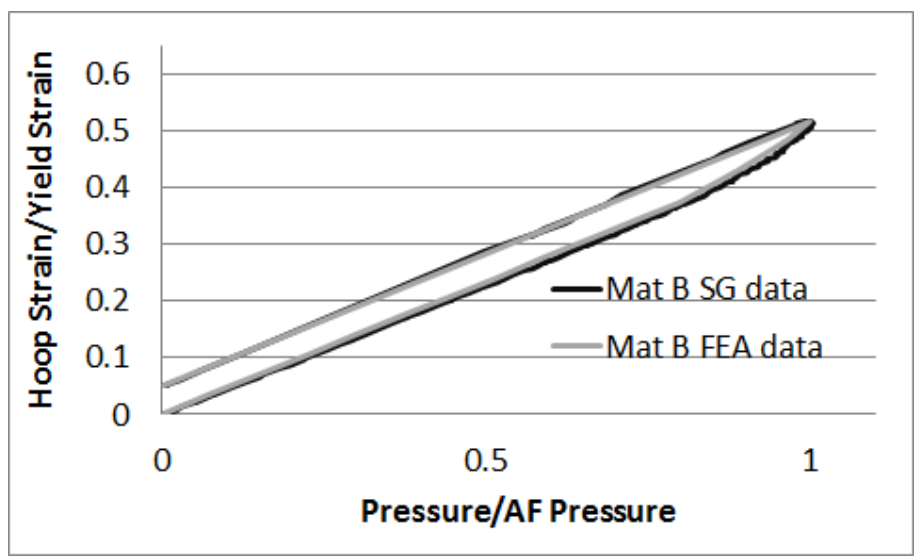

Figure 7: Material B External Gage Comparison.

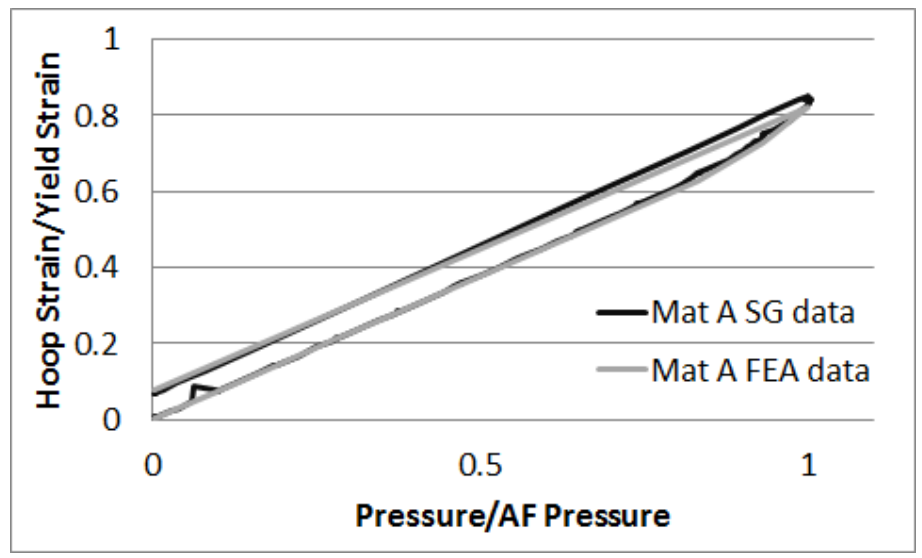

Figure 8: Material A Internal Gage Comparison.

\section{Validation}

As strain readings cannot be taken through the thickness of components, accurate FEA models are of vital importance in predicting behaviour. Internal and external strain gage readings were taken and compared with those predicted by FEA, Figure 5 .

The strain readings from the external gages positioned 90 degrees about the axial axis showed slight differences, likely due to the slight anisotropy of the material. Subsequently the material properties found from tensile testing in each plane were used as material properties during validation simulations.

The final dimensions of FEA model were modified to fully match the actual pressure vessels created. Using both forms of material model, as well as the 


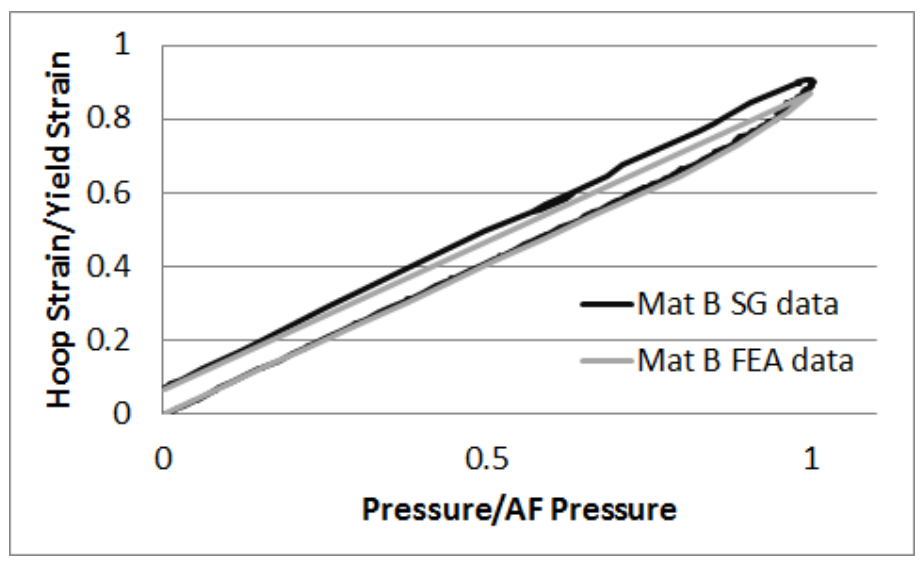

Figure 9: Material B Internal Gage Comparison.

different directional material properties found through tensile testing, the strains present within the appropriate directions at the strain gage rosette locations were compared with those of the actual strain gage readings, Tables 1 and 2.

A direct comparison was made between the external strain gage readings and those predicted from the FEA model, Figures 6 and 7; whilst the internal gages underwent hydrostatic effect correction using correction factors found from the hydrostatic test pieces, Figures 8 and 9.

Comparisons were made between the different hydrostatic correction factors found during the different loading scenarios, from the different pressure vessels, as well as the correction factors calculated through a number of combinations of these data sets.

Although when compared to the strain values at maximum loading, these differences in strain values appear negligible, the scale remains the same upon unloading due to the induced plasticity, thus increasing their \% error.

\section{Conclusions}

The experiment outlined here was successful in quantifying a hydrostatic correction factor up to pressures commonly used within autofrettage to a higher degree of accuracy than has been previously calculated. Comparisons between the different pressure vessels as well as between the different load cases successfully verified the correction factor.

FEA results compared well with their corresponding strain gage readings. Internal strain readings were corrected for the hydrostatic correction factors found during the experiment and compared well with FEA results, and the external gage readings compared even more favourably. During the loading and loaded cases, error values remained low, with the largest errors being $3.8 \%$ and $1.9 \%$ for the internal and external gages respectively. Further work is still required for accu- 
rate residual strain measurement as the unloaded case errors remained as high as $16.7 \%$.

\section{Acknowledgements}

Authors would like to thank Weir SPM, Fort Worth and Mike Herring for all of their help in making this research work successful; as well as the use of ANSYS Inc. software through an Academic research license.

\section{References}

[1] Withers, P.J., Residual stress and its role in failure. Reports on Progress in Physics, 70(12), pp. 2211-2264, 2007.

[2] Thumser, R. \& Bergmann, J.W., Residual stress fields and fatigue analysis of autofrettaged parts. International Journal of Pressure, 79, pp. 113-117, 2002.

[3] Milella, P.P., Stress-based fatigue analysis high cycle fatigue (Chapter 5). Fatigue and Corrosion in Metals, Springer Milan: Milano, pp. 245-308, 2013.

[4] ASME, Boiler and Pressure Vessel Code VIII, Div. 3, 2010.

[5] Optimum autofrettage pressure in thick cylinders. Jurnal Mekanikal, (24), pp. 1-14, 2007.

[6] Withers, P.J. \& Bhadeshia, H.K.D.H., Residual Stress. Part 1 - Measurement techniques. Materials Science and Technology, 17(April), pp. 355-365, 2001.

[7] Monatague, P., The Effect of Hydrostatic Pressures on the Behaviour of Foil Strain Gauges. Strain, 2(2), pp. 17-22, 1966.

[8] Hanafee, J.E., Effect of High Pressure On a Strain Gauge Load Cell. Review of Scientific Instruments, 38(3), p. 328, 1967.

[9] Withers, P. \& Bhadeshia, H.K.D.H., Residual Stress. Part 2 - Nature and origins. Materials Science and Technology, 2001. 